\title{
Direct Method for Solving a Transmission \\ Problem with a Discontinuous Coefficient and the Dirac Distribution
}

\author{
Hideyuki Koshigoe \\ Urban Environment System \\ Chiba University \\ 1-33 Yayoi, Inage \\ 263-8522, Japan \\ koshigoe@tu.chiba-u.ac.jp
}

\begin{abstract}
We construct finite difference solutions of a transmission problem with a discontinuous coefficient and the Dirac distribution by the direct method which we call the successive elimination of lines and then show that the limit function of them satisfies the transmission equation in the sense of distribution.
\end{abstract}

\section{Introduction}

This paper is devoted to the construction and the convergence of finite difference solutions based on the direct method coupled with the fictitious domain method ([2],[9])and distribution theoretical argument ([1]).

Let $\Omega$ be a rectangular domain in $R^{2}, \Omega_{1}$ be an open subset of $\Omega$ and $\Omega_{2}=\Omega \backslash \overline{\Omega_{1}}$, the interface of them be denoted by $\Gamma\left(=\overline{\Omega_{1}} \cap \overline{\Omega_{2}}\right)$ and $\Gamma$ be of class $C^{1}$. The transmission problem considered here is the followings.

Problem I. For $f \in L^{2}(\Omega), \sigma \in L^{2}(\Gamma)$ and $g \in H^{1 / 2}(\partial \Omega)$, find $u \in H^{1}(\Omega)$ such that

$$
\begin{gathered}
-\operatorname{div}(a(x, y) \nabla u)=f+\sigma \delta_{\Gamma} \quad \text { in } D^{\prime}(\Omega), \\
u=g \text { on } \partial \Omega .
\end{gathered}
$$

Here we assume that the discontinuous function $a$ is given by

$$
a(x, y)=\epsilon_{1} \chi_{\Omega_{1}}(x, y)+\epsilon_{2} \chi_{\Omega_{2}}(x, y),
$$

where $\epsilon_{i}>0$ is a parameter $(i=1,2)$ and $\chi_{\Pi}$ is defined by

$$
\chi_{\Pi}(x, y)= \begin{cases}1 & \text { if }(x, y) \in \Pi \\ 0 & \text { if }(x, y) \notin \Pi\end{cases}
$$

for any subset $\Pi$ of $\Omega$.

Equations (1) of this type are arisen in various contexts. One of such examples can be found in the context of electricity and $\left\{\epsilon_{1}, \epsilon_{2}\right\}$ is corresponding to the 
dielectric constant of the material $\left\{\Omega_{1}, \Omega_{2}\right\}$.

We now notice that Problem I is equivalent to the following problem II.

Problem II. Find $\lambda \in H^{1 / 2}(\Gamma)$ and $\left\{u_{1}(\lambda), u_{2}(\lambda)\right\} \in H^{1}\left(\Omega_{1}\right) \times H^{1}\left(\Omega_{2}\right)$ such that

$$
\begin{gathered}
-\epsilon_{1} \triangle u_{1}(\lambda)=f \quad \text { in } \Omega_{1}, \\
-\epsilon_{2} \triangle u_{2}(\lambda)=f \quad \text { in } \Omega_{2}, \\
u_{1}(\lambda)=u_{2}(\lambda)=\lambda \quad \text { on } \Gamma, \\
u_{2}(\lambda)=g \quad \text { on } \partial \Omega,
\end{gathered}
$$

and

$$
\epsilon_{1} \frac{\partial u_{1}(\lambda)}{\partial \nu}-\epsilon_{2} \frac{\partial u_{2}(\lambda)}{\partial \nu}=\sigma \quad \text { on } \Gamma .
$$

Here $\nu$ is the unit normal vector on $\Gamma$ directed from $\Omega_{1}$ to $\Omega_{2}$. Hence introducing the Dirichlet-Neumann map $T$ defined by

$$
T: H^{1 / 2}(\Gamma) \ni \lambda \rightarrow \epsilon_{1} \frac{\partial u_{1}(\lambda)}{\partial \nu}-\epsilon_{2} \frac{\partial u_{2}(\lambda)}{\partial \nu} \in H^{-1 / 2}(\Gamma),
$$

Problem $\mathrm{I}$ is reduced to find $\lambda$ satisfying

$$
T \lambda=\sigma
$$

From this point of view, the purpose of this paper is to show how to solve (8) directly.

This paper is organized as follows. Section 2 describes the finite difference approximation of Problem I. Section 3 is devoted to our numerical algorithm from the viewpoint of the successive elimination of lines coupled with the geometry of domains $\Omega_{1}$ and $\Omega_{2}$. In Sect. 4 , we shall prove the justification of the finite difference scheme and finally discuss the convergence of approximate solutions constructed in Section 3.

\section{Finite Difference Approximation of Problem I}

Without loss of generality we assume that $g=0$ and that $\Omega$ is the unit square in $R^{2}$, i.e., $\Omega=\{(x, y) \mid 0<x, y<1\}$. Let $h \in R$ be a mesh size such that $h=1 / n$ for an integer $n$ and set $\Delta x=\Delta y=h$. We associate with it the set of the grid points:

$$
\begin{aligned}
& \bar{\Omega}_{h}=\left\{P_{i, j} \in R^{2} \mid P_{i, j}=(i h, j h), 0 \leq i, j \leq n\right\} \\
& \Omega_{h}=\left\{P_{i, j} \in R^{2} \mid P_{i, j}=(i h, j h), 1 \leq i, j \leq n-1\right\} .
\end{aligned}
$$

With each grid point $P_{i, j}$ of $\bar{\Omega}_{h}$, we associate the panel $\omega_{i, j}^{0}$ with center $P_{i, j}$ :

$$
\omega_{i, j}^{0} \equiv((i-1 / 2) h,(i+1 / 2) h] \times((j-1 / 2) h,(j+1 / 2) h],
$$


and the cross $\omega_{i, j}^{1}$ with center $P_{i, j}$ :

$$
\omega_{i, j}^{1}=\omega_{i+1 / 2, j}^{0} \cup \omega_{i-1 / 2, j}^{0} \cup \omega_{i, j+1 / 2}^{0} \cup \omega_{i, j-1 / 2}^{0}
$$

where $e_{i}$ denotes the $i$ th unit vector in $R^{2}$ and we set

$$
\omega_{i \pm 1 / 2, j}^{0}=\omega_{i, j}^{0} \pm \frac{h}{2} e_{1}, \quad \omega_{i, j \pm 1 / 2}^{0}=\omega_{i, j}^{0} \pm \frac{h}{2} e_{2}
$$

Moreover using the datum in Problem I, we define

$$
\left\{\begin{array}{llll}
a_{i, j}^{E}=\frac{1}{\Delta x \Delta y} \int_{\omega_{i+1 / 2, j}^{0}} \quad a(x, y) d x d y, & a_{i, j}^{W}=\frac{1}{\Delta x \Delta y} \int_{\omega_{i-1 / 2, j}^{0}} & a(x, y) d x d y, \\
a_{i, j}^{N}=\frac{1}{\Delta x \Delta y} \int_{\omega_{i, j+1 / 2}^{0}} a(x, y) d x d y, & a_{i, j}^{S}=\frac{1}{\Delta x \Delta y} \int_{\omega_{i, j-1 / 2}^{0}} & a(x, y) d x d y, \\
f_{i, j}=\frac{1}{\Delta x \Delta y} \int_{\omega_{i, j}^{0}} f(x, y) d x d y, & \sigma_{i, j}=\frac{1}{\Delta l_{i, j}} \int_{\Gamma \cap \omega_{i j}^{0}} & \sigma(s) d s, \\
\Delta l_{i, j}=\int_{\Gamma \cap \omega_{i, j}^{0}} d s . & &
\end{array}\right.
$$

We then propose the discrete equation of Problem I as follows.

Problem F. Find $\left\{u_{i, j}\right\}(1 \leq i, j \leq n-1)$ such that

$$
\begin{array}{r}
-\frac{1}{\Delta x}\left(a_{i, j}^{E} \frac{u_{i+1, j}-u_{i j}}{\Delta x}-a_{i, j}^{W} \frac{u_{i j}-u_{i-1, j}}{\Delta x}\right) \\
-\frac{1}{\Delta y}\left(a_{i, j}^{N} \frac{u_{i, j+1}-u_{i, j}}{\Delta y}-a_{i, j}^{S} \frac{u_{i, j}-u_{i, j-1}}{\Delta y}\right) \\
=f_{i, j}+\frac{\Delta l_{i, j}}{\Delta x \Delta y} \sigma_{i, j}, \quad 1 \leq i, j \leq n-1 .
\end{array}
$$

Remark 1. The construction of solutions of Problem F will be discussed section 3. Then introducing the base function $\theta_{i, j}$ :

$$
\theta_{i, j}(x, y)= \begin{cases}1, & (x, y) \in \omega_{i, j}^{0} \\ 0, & (x, y) \notin \omega_{i, j}^{0}\end{cases}
$$

we define the piecewise functions $\sigma_{h}$ and $u_{h}$ by

$$
\begin{aligned}
& \sigma_{h}=\sum_{i, j=1}^{n-1} \frac{\Delta l_{i, j}}{\Delta x \Delta y} \sigma_{i, j} \theta_{i, j}(x, y), \\
& u_{h}=\sum_{i, j=1}^{n-1} u_{i, j} \theta_{i, j}(x, y)
\end{aligned}
$$

respectively. In section 4 we shall show that

(i) $\sigma_{h} \rightarrow \sigma \cdot \delta_{\Gamma}$ in $D^{\prime}(\Omega)$,

(ii) $u_{h} \rightarrow u$ weakly in $L^{2}(\Omega), u \in H^{1}(\Omega)$, and

(iii) $u$ is the solution of Problem $\mathrm{I}$ in the sense of distrubution. 


\section{Construction of the Solution of (13)}

\subsection{Geometry of Domain and Principle of the Successive Elimination of Lines}

In this subsection we deal with the $(n-1)$ vectors $\left\{U_{i}\right\}$ instead of the $(n-1)^{2}$ unknowns $u_{i, j}$. For each $i$, set $U_{i}={ }^{t}\left[u_{i, 1}, u_{i, 2}, \cdots, u_{i, n-1}\right](1 \leq i \leq n-1)$. From the equations (13), it follows that

$$
\begin{aligned}
& \left(a_{i, j}^{W}+a_{i, j}^{E}+a_{i, j}^{S}+a_{i, j}^{N}\right) u_{i, j}-a_{i, j}^{S} u_{i, j-1}-a_{i, j}^{N} u_{i, j+1} \\
& \quad=a_{i, j}^{W} u_{i-1, j}+a_{i, j}^{E} u_{i+1, j}+(\Delta x)^{2} f_{i, j}+\sigma_{i, j} \cdot \Delta l_{i, j}
\end{aligned}
$$

Now fix $i(1 \leq i \leq n-1)$. Paying attention to the vector $U_{i}$ in (15) and setting $a_{i, j}^{\epsilon}=a_{i, j}^{W}+a_{i, j}^{E}+a_{i, j}^{S}+a_{i, j}^{N}$, Problem F w.r.t. $\left\{u_{i, j}\right\}$ is reduced to Problem M w.r.t. $\left\{U_{i}\right\}$.

Problem M. Find $U_{i}(1 \leq i \leq n-1)$ satisfying

$$
A_{i}^{\epsilon} U_{i}=A_{i}^{W} U_{i-1}+A_{i}^{E} U_{i+1}+F_{i} \quad(1 \leq i \leq n-1)
$$

where $U_{0}=0, U_{n}=0, F_{i}$ is given by the data $\{f, \sigma\}, A_{i}^{\epsilon}$ is a tridiagonal matrix defined by

$$
A_{i}^{\epsilon}=\left(\begin{array}{ccccccc}
a_{i, 1}^{\epsilon} & -a_{i, 1}^{N} & 0 & \cdots & \cdots & \cdots & 0 \\
-a_{i, 2}^{S} & a_{i, 2}^{\epsilon} & -a_{i, 2}^{N} & 0 & \vdots & \vdots & \vdots \\
0 & \ddots & \ddots & \ddots & 0 & \vdots & \vdots \\
\vdots & 0 & \ddots & \ddots & \ddots & 0 & \vdots \\
\vdots & \vdots & 0 & \ddots & \ddots & \ddots & 0 \\
\vdots & \vdots & \vdots & 0 & -a_{i, n-2}^{S} & a_{i, n-2}^{\epsilon} & -a_{i, n-2}^{N} \\
0 & \cdots & \ldots & \ldots & 0 & -a_{i, n-1}^{S} & a_{i, n-1}^{\epsilon}
\end{array}\right)
$$

and $A_{i}^{W}, A_{i}^{E}$ are the diagonal matrices given by

$$
A_{i}^{W}=\operatorname{diag}\left[a_{i, j}^{W}\right]_{1 \leq j \leq n-1} \text { and } A_{i}^{E}=\operatorname{diag}\left[a_{i, j}^{E}\right]_{1 \leq j \leq n-1}
$$

Remark 2. For each $i(1 \leq i \leq n-1), A_{i}^{\epsilon}$ is a symmetric matrix.

In fact, $a_{i, j}^{N}=a_{i, j+1}^{S}$ holds from the definition (12).

Moreover in order to reduce the numbers of equations of Problem M, we separate unknown vector $U_{i}$ into two parts considering the geometry of the domain $\Omega$ and the interface $\Gamma$. We first introduce the set of interface lattice points $\Gamma_{h}$ and boundary lattice points $\partial \Omega_{h}$ as follows;

(i) $\Gamma_{h}=\left\{P_{i, j}=(i h, j h) \mid \Gamma \cap \omega_{i, j}^{1} \neq \emptyset\right\}$,

(ii) $\partial \Omega_{h}=\bar{\Omega}_{h} \backslash \Omega_{h}$. 


\section{Division of the Unknown Vector $\left\{U_{i}\right\}$}

For each $U_{i}=\left\{u_{i, j}\right\}_{1 \leq j \leq n-1}$, we define $U_{i}^{\prime}=\left\{u_{i, j}^{\prime}\right\}_{1 \leq j \leq n-1}$ and $W_{i}=\left\{w_{i, j}\right\}_{1 \leq j \leq n-1}$ as follows;

$$
u_{i, j}^{\prime}=\left\{\begin{array}{ll}
0 & \text { if } P_{i, j} \in \Gamma_{h} \\
u_{i, j} & \text { if } P_{i, j} \in \Omega_{h} \backslash \Gamma_{h},
\end{array} \quad w_{i, j}= \begin{cases}u_{i, j} & \text { if } P_{i, j} \in \Gamma_{h} \\
0 & \text { if } \quad P_{i, j} \in \Omega_{h} \backslash \Gamma_{h}\end{cases}\right.
$$

and devide $U_{i}$ into two parts by

$$
U_{i}=U_{i}^{\prime}+W_{i}
$$

We then introduce the new vector $\left\{V_{i}\right\}$ defined by

$$
V_{i}=A_{i}^{W} U_{i}^{\prime}\left(=A_{i}^{E} U_{i}^{\prime}\right) \quad(1 \leq i \leq n-1) .
$$

From the definition of $\left\{U_{i}^{\prime}\right\}$ and $\left\{V_{i}\right\}$, we get

Lemma 1. $A_{i}^{\epsilon} U_{i}^{\prime}=B V_{i}, A_{i}^{w} U_{i-1}^{\prime}=V_{i-1}$ and $A_{i}^{E} U_{i+1}^{\prime}=V_{i+1}$ hold $\quad(1 \leq i \leq$ $n-1)$. Here $B$ is a block tridiagonal matrix in the discretization of the Laplace operator in $\Omega$ with homogeneous Dirichlet boundary conditions. i.e., $B=\left[b_{i j}\right]$ is an $(n-1) \times(n-1)$ tridiagonal matrix such that $B=\operatorname{tridiag}[-1,4,-1]$.

Therefore the following equations are derived from Problem M, (17)-(21) and Lemma 1.

Problem PN. Find $\left\{V_{i}, W_{i}\right\}$ such that for $i(1 \leq i \leq n-1)$,

$$
B V_{i}=V_{i-1}+V_{i+1}+F_{i}+\left(A_{i}^{W} W_{i-1}-A_{i}^{\epsilon} W_{i}+A_{i}^{E} W_{i+1}\right)
$$

where $V_{0}=V_{n}=W_{0}=W_{n}=0$.

Moreover in order to deduce the equation of $\left\{W_{i}\right\}$ from Problem PN, we review the princple of the successive elimination of lines. The following proposition 1 was proved under two assumptions

Assumption 1. Let $B=\operatorname{tridiag}[-1,4,-1] \in \mathrm{R}^{(\mathrm{n}-1) \times(\mathrm{n}-1)}$.

Assumption 2. Let $X_{i}$ and $Y_{i} \in R^{(n-1)}$ be satisfying the equations of the form : $B X_{i}=X_{i-1}+X_{i+1}+Y_{i} \quad(1 \leq i \leq n-1)$.

Proposition 1. Under the above assumptions, $X_{k}(1 \leq k \leq n-1)$ is directly determined by

$$
Q X_{k}=\sum_{i=1}^{k-1} D_{n-k, i} Q Y_{i}+\sum_{i=k}^{n-1} D_{k, n-i} Q Y_{i}
$$

where each $D_{l, i}(1 \leq l, i \leq n-1)$ is a diagonal matrix defined by

$$
\begin{aligned}
D_{l, i} & =\operatorname{diag}\left[\left(\sinh \left(l \lambda_{j}\right) \sinh \left(i \lambda_{j}\right)\right) /\left(\sinh \left(n \lambda_{j}\right) \sinh \left(\lambda_{j}\right)\right)\right]_{1 \leq j \leq n-1} \\
\lambda_{j} & =\operatorname{arccosh}(2-\cos (j \pi / n)),
\end{aligned}
$$


and $Q\left(=\left(q_{i, j}\right)_{1 \leq i, j \leq n-1}\right)$ is the othogonal matrix such that

$$
q_{i, j}=\sqrt{\frac{2}{n}} \sin \left(\frac{i j \pi}{n}\right) \quad(1 \leq i, j \leq n-1) .
$$

Remark 3. We call this proposition the princile of the successive elimination of lines (see also [6], [7], [11]).

Remark 4. Set $Q_{i}={ }^{t}\left(q_{i, 1}, q_{i, 2}, \cdots \cdots, q_{i, n-1}\right) \quad(1 \leq i \leq n-1)$. Then $\left\{Q_{i}\right\}_{1 \leq i \leq n-1}$ is the orthonormal system, which is used in the next subsection.

\subsection{Numerical Algorithm}

In this subsection, we show our numerical algorithm by use of the principle of the successive elimination of lines. First applying directly Proposition 1 to Problem $\mathrm{PN}$, we have

Lemma 2. Problem $P N$ is equivalent to find $\left\{V_{k}, W_{k}\right\}(1 \leq k \leq n-1)$ satisfying

$$
\begin{aligned}
Q V_{k} & =\sum_{i=1}^{k-1} D_{n-k, i} Q\left(A_{i}^{W} W_{i-1}-A_{i}^{\epsilon} W_{i}+A_{i}^{E} W_{i+1}\right) \\
& +\sum_{i=k}^{n-1} D_{k, n-i} Q\left(A_{i}^{W} W_{i-1}-A_{i}^{\epsilon} W_{i}+A_{i}^{E} W_{i+1}\right) \\
& +\left(\sum_{i=1}^{k-1} D_{n-k, i} Q F_{i}+\sum_{i=k}^{n-1} D_{k, n-i} Q F_{i}\right) .
\end{aligned}
$$

Using the orthogonal property of $Q$ and the definitions of $V_{k}$ and $\Gamma_{h}$, we get

$$
{ }^{t} Q_{l} Q V_{k}=0 \text { for any } l \text { such that } P_{k, l} \in \Gamma_{h},
$$

from which it follows

Lemma 3. $\left\{W_{i}\right\}_{1 \leq i \leq n-1}$ in (26) satisfies the equations (27):

$$
\begin{aligned}
& \sum_{i=1}^{k-1}{ }^{t} Q_{l} D_{n-k, i} Q\left(-A_{i}^{W} W_{i-1}+A_{i}^{\epsilon} W_{i}-A_{i}^{E} W_{i+1}\right) \\
& +\sum_{i=k}^{n-1}{ }^{t} Q_{l} D_{k, n-i} Q\left(-A_{i}^{W} W_{i-1}+A_{i}^{\epsilon} W_{i}-A_{i}^{E} W_{i+1}\right) \\
& ={ }^{t} Q_{l}\left(\sum_{i=1}^{k-1} D_{n-k, i} Q F_{i}+\sum_{i=k}^{n-1} D_{k, n-i} Q F_{i}\right)
\end{aligned}
$$

for $(k, l)$ such that $P_{k, l} \in \Gamma_{h}$.

Conversely one may have a question whether it is possible to construct $\left\{V_{k}, W_{k}\right\}$ uniquely satisfying (26) from the equation (27). But the answer is yes and we shall prove it in the next section as the following theorem.

Theorem 1. There exists a unique solution $\left\{W_{i}\right\}_{1 \leq i \leq n-1}$ of the linear system (27).

Hence the remainder part $\left\{V_{k}\right\}_{1 \leq k \leq n-1}$ of $\left\{U_{i}\right\}_{1 \leq i \leq n-1}$ is automatically computed by Theorem 1 and Lemma 2 . i.e., 
Theorem 2. $\quad V_{k}$ is determined by

$$
\begin{aligned}
v_{k, l} & =\sum_{i=1}^{k-1}{ }^{t} Q_{l} D_{n-k, i} Q\left(A_{i}^{W} W_{i-1}-A_{i}^{\epsilon} W_{i}+A_{i}^{E} W_{i+1}\right) \\
+ & \sum_{i=k}^{n-1}{ }^{t} Q_{l} D_{k, n-i} Q\left(A_{i}^{W} W_{i-1}-A_{i}^{\epsilon} W_{i}+A_{i}^{E} W_{i+1}\right) \\
+ & { }^{t} Q_{l}\left(\sum_{i=1}^{k-1} D_{n-k, i} Q F_{i}+\sum_{i=k}^{n-1} D_{k, n-i} Q F_{i}\right) .
\end{aligned}
$$

for $(k, l)$ such that $P_{k, l} \in \Omega_{h} \backslash \Gamma_{h}$.

Therefore we summarize our numerical algorithm.

\section{Numerical Algorithm}

1st step: Calculate the solution $\left\{W_{i}\right\}$ on $\Gamma_{h}$ of (27).

2nd step: Compute $\left\{V_{k}\right\}$ on $\Omega_{h} \backslash \Gamma_{h}$ by use of the formulation in Theorem 2 .

\section{Convergence of Approximate Solutions}

\subsection{Function Space $V_{h}$}

In order to justify our numerical scheme(13), we first define the piecewise function $\theta_{\alpha, \beta} \quad(0 \leq \alpha, \beta \leq n)$ as follows;

$\theta_{\alpha, \beta}(x, y)=\theta(x-\alpha h, y-\beta h)$ where $\theta(x, y)= \begin{cases}1, & (x, y) \in \omega_{0,0}^{0} \\ 0, & (x, y) \notin \omega_{0,0}^{0},\end{cases}$

and $\theta_{0, j}=\theta_{n, j}=\theta_{i, 0}=\theta_{i, n}=0(i, j=1, \cdots, n)$. We then introduce the function space $V_{h}$ generated by $\theta_{i, j}$. i.e., $\phi \in V_{h}$, is of the form:

$$
\phi(x, y)=\sum_{i, j=1}^{n-1} \phi_{i, j} \quad \theta_{i, j}(x, y), \quad \phi_{i, j} \in R .
$$

We now introduce the following approximation $\left\{\delta_{h}^{1}, \delta_{h}^{2}, \nabla_{h},(\operatorname{div})_{h}\right\}$ of $\{\partial / \partial x$, $\partial / \partial y, \nabla, \operatorname{div}\}$.

(i) $\delta_{h}^{1}, \delta_{h}^{2}: L^{\infty}\left(R^{2}\right) \rightarrow L^{\infty}\left(R^{2}\right)$ are defined by

$$
\begin{aligned}
& \left(\delta_{h}^{1} u\right)(x, y)=\frac{1}{h}\left(u\left(x+\frac{1}{2} h, y\right)-u\left(x-\frac{1}{2} h, y\right)\right), \\
& \left(\delta_{h}^{2} u\right)(x, y)=\frac{1}{h}\left(u\left(x, y+\frac{1}{2} h\right)-u\left(x, y-\frac{1}{2} h\right)\right) .
\end{aligned}
$$

(ii) $\nabla_{h}: L^{\infty}\left(R^{2}\right) \rightarrow\left(L^{\infty}\left(R^{2}\right)\right)^{2}$ is defined by

$$
\left(\nabla_{h} u\right)(x, y)=\left(\left(\delta_{h}^{1} u\right)(x, y),\left(\delta_{h}^{2} u\right)(x, y)\right) .
$$

(iii) (div) $)_{h}:\left(L^{\infty}\left(R^{2}\right)\right)^{2} \rightarrow L^{\infty}\left(R^{2}\right)$ is defined by

$$
(\operatorname{div})_{h}(u(x, y), v(x, y))=\left(\delta_{h}^{1} u\right)(x, y)+\left(\delta_{h}^{2} v\right)(x, y)
$$

for $u, v \in L^{\infty}\left(R^{2}\right)$.

Then the norm $\|\cdot\|$ in $V_{h}$ is equipped as follows;

$$
\|u\|=\sqrt{\|u\|_{L^{2}(\Omega)}^{2}+\left\|\nabla_{h} u\right\|_{L^{2}(\Omega)}^{2}} \text { for } u \in V_{h},
$$

from which we get 
Lemma 4. (i) $V_{h}$ is a Hilbert space.

(ii) $\left(\delta_{h}^{i} u, \phi\right)_{L^{2}(\Omega)}=-\left(u, \delta_{h}^{i} \phi\right)_{L^{2}(\Omega)} \quad$ for $u, \phi \in V_{h} \quad(i=1,2)$.

Furthermore using the notations $\left\{a_{i, j}^{W}, a_{i, j}^{S}, f_{i, j}, \Delta l_{i, j}, \sigma_{i, j}\right\}$ in (12), we define approximate functions of $a, f$ and $\sigma$ respectively as follows:

$$
\begin{aligned}
a_{h}^{W}(x, y) & =\sum_{j=1}^{n-1} \sum_{i=1}^{n} a_{i, j}^{W} \theta_{i-1 / 2, j}(x, y), \\
a_{h}^{S}(x, y) & =\sum_{i=1}^{n-1} \sum_{j=1}^{n} \quad a_{i, j}^{S} \theta_{i, j-1 / 2}(x, y), \\
f_{h}(x, y) & =\sum_{i, j=1}^{n-1} f_{i, j} \theta_{i, j}(x, y), \\
\sigma_{h}(x, y) & =\sum_{i, j=1}^{n-1} \frac{\Delta l_{i, j}}{\Delta x \Delta y} \sigma_{i, j} \theta_{i, j}(x, y) .
\end{aligned}
$$

\subsection{Approximate Solution in $V_{h}$ of Problem I}

In this subsection the approximate solution in $V_{h}$ for Problem I is considered. We first propose the following approximation of Problem I in $V_{h}$.

Problem V. Find $u_{h} \in V_{h}$ such that

$$
\begin{array}{r}
-(\operatorname{div})_{h}\left(a_{h}^{W}(x, y)\left(\delta_{h}^{1} u_{h}\right), a_{h}^{S}(x, y)\left(\delta_{h}^{2} u_{h}\right)\right)(x, y) \\
=f_{h}(x, y)+\sigma_{h}(x, y) \text { for }(x, y) \in \bigcup_{i, j=1}^{n-1} \omega_{i, j}^{0} .
\end{array}
$$

We then get a following relation between Problem F and Problem V.

Lemma 5. Problem $F$ and Problem $V$ are equivalent.

Proof. Using the notations in 4.1 and the property of the support for piecewise functions, the equation (33) is of the form

$$
\begin{aligned}
& -\sum_{i, j=1}^{n-1}\left(\frac{1}{\Delta x}\left(a_{i, j}^{E} \frac{u_{i+1, j}-u_{i j}}{\Delta x}-a_{i, j}^{W} \frac{u_{i j}-u_{i-1, j}}{\Delta x}\right)\right. \\
& \left.+\frac{1}{\Delta y}\left(a_{i, j}^{N} \frac{u_{i, j+1}-u_{i j}}{\Delta y}-a_{i, j}^{S} \frac{u_{i j}-u_{i, j-1}}{\Delta y}\right)\right) \theta_{i, j}(x, y) \\
& =\sum_{i, j=1}^{n-1}\left(f_{i, j}+\frac{\Delta l_{i, j}}{\Delta x \Delta y} \sigma_{i, j}\right) \theta_{i, j}(x, y)
\end{aligned}
$$

for $(x, y) \in \bigcup_{i, j=1}^{n-1} \omega_{i, j}^{0}$. Hence this lemma holds.

Using the discrete Poincaré inequality and the trace theorem ([5]), we get

Proposition 2. There exists a unique function $u_{h} \in V_{h}$ satisfying (33).

The uniqueness of $\left\{W_{i}\right\}$ in (27) is now proved.

Proof of Theorem 1. Assume that there are two solutions $\left\{W_{i}\right\}$ and $\left\{\widetilde{W}_{i}\right\}$ satisfying the linear system (27). Then from Lemma 2, and (19)-(21), there are two solutions $\left\{U_{i}\right\}$ and $\left\{\widetilde{U}_{i}\right\}$ of Problem F. But this is contradictory to Proposition 2 by use of Lemma 5. Therefore the unique existence of the solution $\left\{W_{i}\right\}$ is ensured. 


\subsection{Convergence Theorem}

We proceed to discuss the convergence of $\left\{u_{h}\right\}$.

Theorem 3. (i) There exists $u \in H_{0}^{1}(\Omega)$ such that $u_{h} \rightarrow u$ weakly in $L^{2}(\Omega)$. (ii) $u$ satisfies that for any $\phi \in D(\Omega)$,

$$
\langle-\operatorname{div}(\mathrm{a} \nabla \mathrm{u}), \phi\rangle_{\mathrm{D}^{\prime}(\Omega)}=(\mathrm{f}, \phi)_{\mathrm{L}^{2}(\Omega)}+(\sigma, \phi)_{\mathrm{L}^{2}(\Gamma)}
$$

Proof. We divide the proof into four steps.

Step 1. There exists a subsequence $u_{h}$, also denoted by $u_{h}$, such that $u_{h} \rightarrow u$ weakly in $L^{2}(\Omega)$ and $\nabla_{h} u_{h} \rightarrow \nabla u$ weakly in $L^{2}(\Omega)$.

In fact, it follows from the bilinear form of (33) in $V_{h}$ and the discrete Poincaré inequality.

Step 2. $f_{h} \rightarrow f$ in $L^{2}(\Omega)$ and $a_{h}^{W} \rightarrow a$ a.e. in $\Omega, \quad a_{h}^{S} \rightarrow a \quad$ a.e. in $\Omega$. Because $f \in L^{2}(\Omega)$ and $a$ is continuous in $\Omega \backslash \Gamma$.

Step 3. $\sigma_{h} \rightarrow \sigma \cdot \delta(\Gamma)$ in $D^{\prime}(\Omega)$.

In fact, Set $I \equiv\left\langle\sigma_{h}-\sigma \cdot \delta(\Gamma), \phi\right\rangle$. Then

$$
\begin{aligned}
I & =\sum_{i, j=1}^{n-1} \int_{\omega_{i, j}^{0}} \frac{\Delta l_{i, j}}{h^{2}} \sigma_{i, j} \phi(x, y) d x d y-\int_{\Gamma} \sigma(s) \phi(x(s), y(s)) d s \\
& =\sum_{i, j=1}^{n-1} \int_{\omega_{i, j}^{0} \cap \Gamma} \sigma(s)\left\{\frac{1}{h^{2}} \int_{\omega_{i, j}^{0}} \phi(x, y) d x d y\right\} d s-\int_{\Gamma} \sigma(s) \phi(x(s), y(s)) d s .
\end{aligned}
$$

Since $\phi \in D(\Omega)$, there exists a point $\left(x_{i, j}, y_{i, j}\right)$ in $\omega_{i, j}^{0}$ such that

Hence

$$
\frac{1}{h^{2}} \int_{\omega_{i, j}^{0}} \phi(x, y) d x d y=\phi\left(x_{i, j}, y_{i, j}\right), \quad 1 \leq i, j \leq n-1 \text {. }
$$

$$
\begin{gathered}
|I|=\left|\sum_{i, j=1}^{n-1} \int_{\omega_{i, j}^{0} \cap \Gamma} \sigma(s) \phi\left(x_{i, j}, y_{i, j}\right) d s-\int_{\Gamma} \sigma(s) \phi(x(s), y(s)) d s\right| \\
=\mid \int_{\Gamma} \sigma(s) \sum_{i, j=1}^{n-1}\left[\left(\phi\left(x_{i, j}, y_{i, j}\right)-\phi(x(s), y(s)) \theta_{i, j}(x(s), y(s))\right] d s \mid\right. \\
\leq \quad\left(\int_{\Gamma}|\sigma(s)|^{2} d s\right)^{1 / 2} \quad\left(\int_{\Gamma} \sum_{i, j=1}^{n-1} \mid \phi\left(x_{i, j}, y_{i, j}\right)-\right. \\
\left.\left.\phi(x(s), y(s))\right|^{2} \theta_{i, j}(x(s), y(s)) d s\right)^{1 / 2}
\end{gathered}
$$$$
\leq \quad\left(\int_{\Gamma}|\sigma(s)|^{2} d s\right)^{1 / 2}\left(\sum_{i, j=1}^{n-1} \int_{\omega_{i, j}^{0} \cap \Gamma}\left|\left(x_{i, j}, y_{i, j}\right)-(x(s), y(s))\right|^{2} .\right.
$$

$\left.|\nabla \phi|_{L^{\infty}(\Omega)}^{2} d s\right)^{1 / 2}$

$\leq \sqrt{2 \mu(\Gamma)} h|\sigma|_{L^{2}(\Gamma)} \cdot|\nabla \phi|_{L^{\infty}(\Omega)} \quad$ where $\mu(\Gamma)=\int_{\Gamma} d s$.

This shows the statement of Step 3. 
Step 4. For $\phi \in D(\Omega)$, the equation

$$
-\left\langle\frac{\partial}{\partial x}\left(a \frac{\partial u}{\partial x}\right)+\frac{\partial}{\partial y}\left(a \frac{\partial u}{\partial y}\right), \phi\right\rangle_{D^{\prime}(\Omega)}=(f, \phi)_{L^{2}(\Omega)}+(\sigma, \phi)_{L^{2}(\Gamma)}
$$

holds.

In fact, it follows from Proposition 2 that for sufficiently small $h$,

$$
\begin{aligned}
& \left(-(\operatorname{div})_{h}\left(a_{h}^{W}(x, y)\left(\delta_{1} u_{h}\right), a_{h}^{S}(x, y)\left(\delta_{2} u_{h}\right)\right)(x, y), \phi(x, y)\right)_{L^{2}(\Omega)} \\
& =\left(\left(a_{h}^{W}(x, y)\left(\delta_{1} u_{h}\right)(x, y), a_{h}^{S}(x, y)\left(\delta_{2} u_{h}\right)(x, y)\right), \nabla_{h} \phi(x, y)\right)_{L^{2}(\Omega)} \\
& =\left(f_{h}(x, y)+\sigma_{h}(x, y), \phi(x, y)\right)_{L^{2}(\Omega)}
\end{aligned}
$$

We then use the results from the Step1 to Step 3 and as $h \rightarrow 0$ in the above equation, we have

$$
\left(a \frac{\partial u}{\partial x}, \frac{\partial \phi}{\partial x}\right)_{L^{2}(\Omega)}+\left(a \frac{\partial u}{\partial y}, \frac{\partial \phi}{\partial y}\right)_{L^{2}(\Omega)}=(f, \phi)_{L^{2}(\Omega)}+(\sigma, \phi)_{L^{2}(\Gamma)}
$$

Therefore combining it with the distribution formula, Step 4 is shown.

Finally we are able to conclude that the full sequence $\left\{u_{h}\right\}$ converges weakly to the solution $u$ of Problem I since Problem I has a unique solution in $H^{1}(\Omega)$ as well known fact(cf. [8,10]).

Acknowledgment. The author is grateful to Prof. Kawarada of Chiba University for his significant comments and also wish to thank Prof. Kitahara of Kogakuin University for helpful discussions.

\section{References}

1. H. Fujita,H. Kawahara and H. Kawarada, Distribution theoretical approach to fictitious domain method for Neumann problems, East-West J. Math., vol. 3, no. 2 (1995), 111-126.

2. R. Glowinski, T.W. Pan and J. Periaux, A fictitious domain method for Dirichlet problem and applications, Computer Methods in Applied Mechanics and Engineering 111 (1994), 283-303.

3. H. Han and Z. Huang, The direct method of lines for the numerical solutions of interface problem, Computer methods in Applied Mechanics and Engineering, 171 (1999), 61-75.

4. F. John, Lectures on Advanced Numerical Analysis, Gordon and Breach Science Publishers, Inc., New York (1967).

5. H. Kawarada, Free boundary problem - theory and numerical method, Tokyo University Press (1989)(in Japanese) .

6. Koshigoe, H. and Kitahara, K., "Method of lines coupled with fictitious domain for solving Poisson's equation", Mathematical Sciences and Applications (Gakuto International Series), Vol. 12 (1999), 233-241.

7. Koshigoe, H. and Kitahara, K., "Numerical algorithm for finite difference solutions constructed by fictitious domain and successive eliminations of lines", Japan SIAM, Vol. 10, No. 3 (2000), 211-225 (in Japanese). 
8. J.L. Lions, Optimal control of systems governed by partial differential equations, 170, Springer-Verlarg (1971).

9. G.I. Marchuk,Y.A. Kuznetsov and A.M. Matsokin, Fictitious domain and domain decomposition methods, Sov. J. Numer. Anal. Math. Modelling, Vol. 1, No. 1 (1986) 3-35.

10. S. Mizohata, The theory of partial differential equations, Cambridge at the University Press (1973).

11. K. Nakashima, Numerical computation of elliptic partial differential equations I, Method of Lines, Memoirs of the school of science \& engineering, Waseda Univ., No. 29, 115-127,1965. 